
\title{
BIIBHECa
}

Biblioteca Universitaria

ISSN: 0187-750X

public@dgb.unam.mx

Universidad Nacional Autónoma de México

México

Novelo Peña, Raúl; Sánchez Avillaneda, Ma. del Rocío

La Biblioteca del Instituto de Investigaciones en Matemáticas Aplicadas y en Sistemas (iimas)

Biblioteca Universitaria, vol. 15, núm. 2, julio-diciembre, 2012, pp. 182-194

Universidad Nacional Autónoma de México

Distrito Federal, México

Disponible en: http://www.redalyc.org/articulo.oa?id=28528265008

- Cómo citar el artículo

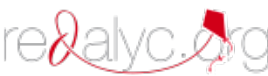

- Número completo

- Más información del artículo

- Página de la revista en redalyc.org

Sistema de Información Científica

Red de Revistas Científicas de América Latina, el Caribe, España y Portugal

Proyecto académico sin fines de lucro, desarrollado bajo la iniciativa de acceso abierto 


\section{La Biblioteca del Instituto de Investigaciones en Matemáticas Aplicadas y en Sistemas (IIMAS)}

\section{Raúl Novelo Peña*, Ma. del Rocío Sánchez Avillaneda**}
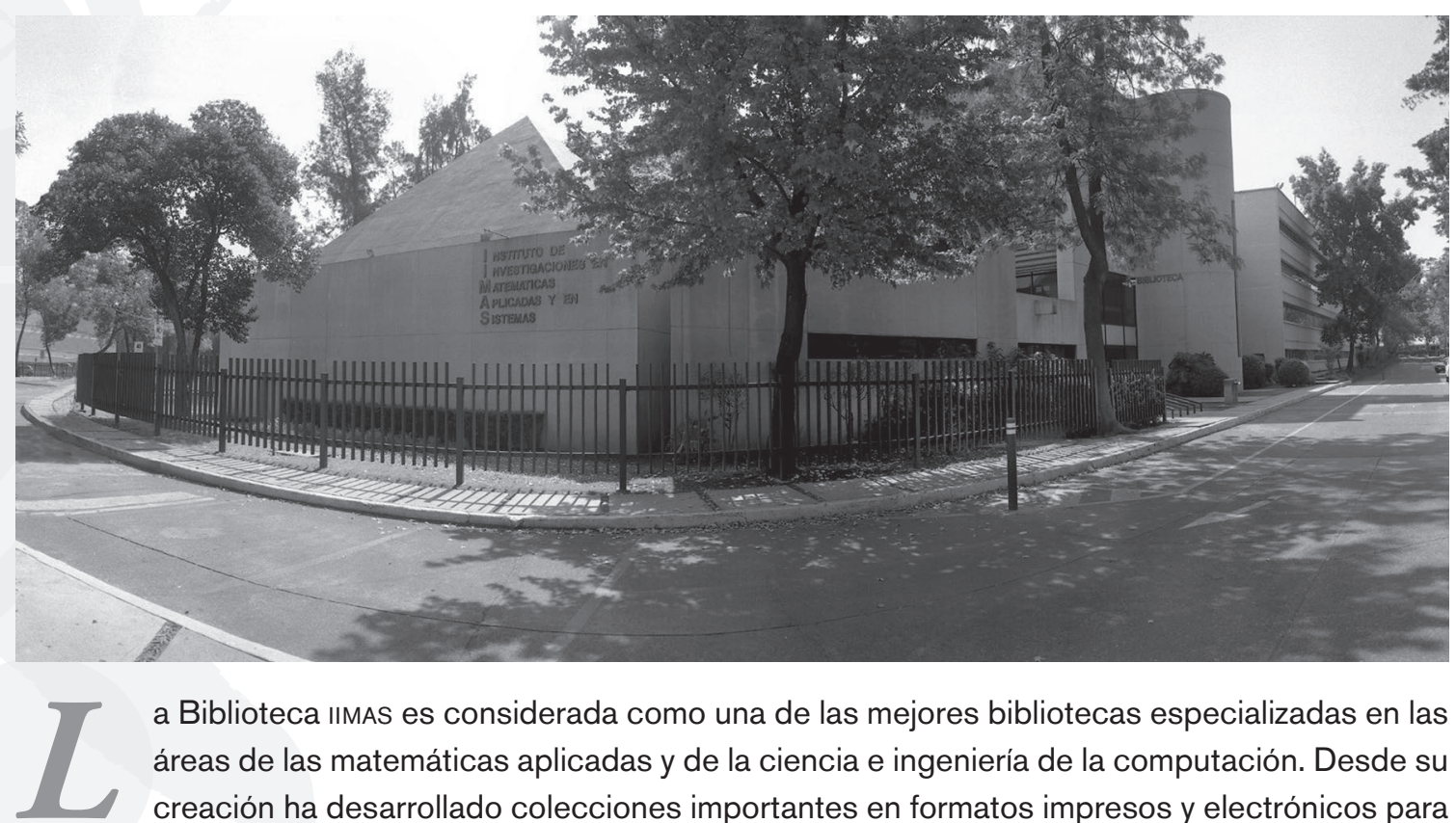

a Biblioteca IIMAS es considerada como una de las mejores bibliotecas especializadas en las áreas de las matemáticas aplicadas y de la ciencia e ingeniería de la computación. Desde su creación ha desarrollado colecciones importantes en formatos impresos y electrónicos para satisfacer las necesidades de información de sus usuarios.

Con el nuevo edificio, la Biblioteca logró una mejor funcionalidad y a partir de su reorganización administrativa en el año 2001 se empezaron a ofrecer nuevos servicios teniendo como ejes principales a sus usuarios y el desarrollo de colecciones electrónicas especializadas.

La automatización de los procesos y servicios dentro de la Biblioteca ha sido una de las tareas principales que se han venido desarrollando a partir del año 2000; a la fecha se cuenta con una página web y se han implementado varios módulos y aplicaciones del sistema ALEPH para facilitar el uso de sus colecciones y agilizar varios de los servicios que se ofrecen.

La Biblioteca ha desarrollado y participado en diferentes actividades que le han permitido mejorar sus servicios y colecciones e incidir en algunos de los avances del sistema bibliotecario de la Universidad.

* Coordinador de la Biblioteca del Instituto de Investigaciones en Matemáticas Aplicadas y en Sistemas (IIMAS), Circuito Interior s/n, unam, C.U., C.P. 04510, México, D.F. México. Correo electrónico: rnovelo@leibniz.iimas.unam.mx

** Biblioteca del Instituto de Investigaciones en Matemáticas Aplicadas y en Sistemas (IIMAS), Circuito Interior s/n, unAM, C.U., C.P. 04510, México, D.F. México. Correo electrónico: marsa@leibniz.iimas.unam.mx 


\section{Antecedentes del IIMAS ${ }^{1}$}

El Instituto de Investigaciones en Matemáticas Aplicadas y en Sistemas (IIMAS) tiene como antecedente el Centro de Cálculo Electrónico (CCE), fundado en junio de 1958 en la Facultad de Ciencias, año en que se instala la primera computadora, una IBM-650, en la Universidad Nacional Autónoma de México (UNAM) y en el país, con el fin de utilizarla para el avance de la ciencia en México. Su director fundador fue el ingeniero Sergio F. Beltrán López durante el periodo de 1958 a 1967.

En 1967 el CCE adquirió otra computadora con tecnología muy avanzada para su tiempo, y su uso se difundió rápidamente entre especialistas y universitarios. Así, al incrementarse sustancialmente las actividades relacionadas con el servicio a los usuarios, se propuso la fusión de la Dirección General de Sistematización de Datos con el CCE para que se transformaran en el Centro de Investigaciones en Matemáticas Aplicadas, Sistemas y Servicios (CIMASS); de tal forma que para finales de 1970 se fundó este nuevo Centro, dependiente de la entonces Coordinación de Ciencias, integrándose por primera vez en la Universidad el apoyo de cómputo en las áreas académica y administrativa, reuniendo bajo una sola dirección los servicios de cómputo para la docencia, la investigación y la administración; además, se dio inicio formalmente con las actividades de investigación, se crearon los departamentos de Matemáticas y Mecánica y el de Estadística, Probabilidad e Investigación de Operaciones, y se participó activamente en la creación y puesta en marcha de la Maestría en Estadística e Investigación de Operaciones en 1972 con la finalidad de formar estadísticos de alto nivel con una sólida formación matemática.

El 15 de marzo de 1973 el CIMASS se dividió en dos centros: el Centro de Servicios de Cómputo (csc), que daría apoyo a la administración y a la academia, y el Centro de Investigaciones en Matemáticas Aplicadas

Universidad Nacional Autónoma de México. Instituto de Investigaciones en Matemáticas Aplicadas y en Sistemas. Acerca del IIMAS [en línea]. <http://www.iimas.unam.mx/iimas/> [Consulta: septiembre 2012]. y en Sistemas (CIMAS), que se dedicaría a la investigación siendo su director el Dr. Tomás Garza Hernández.

A partir de ese año se amplían los grupos de trabajo y se diversifican las actividades; se desarrolló investigación en ciencias de la computación, probabilidad y estadística, en investigación de operaciones, sistemas universitarios, cibernética y aplicación de las matemáticas a problemas sociales, técnicos, científicos, económicos y administrativos, de interés tanto para la UNAM como para el país; se creó en marzo de 1975 la Maestría en Ciencias de la Computación, cuya coordinación y organización académica fueron confiadas al CIMAS; además, se formaron grupos de trabajo con alta productividad, consistencia y madurez, que finalmente condujeron a que el Centro se convirtiera en el Instituto de Investigaciones en Matemáticas Aplicadas y en Sistemas, al ser aprobado por el Consejo Universitario el 10 de marzo de 1976.

En sus inicios como instituto, el IIMAS se centró en el estudio de nuevas áreas que requerían ideas diferentes a las tradicionales, así surgieron dos corrientes de pensamiento: una que trató de responder esas nuevas preguntas en el ámbito de la investigación fundamental buscando formulaciones y soluciones cuantitativas y una segunda que buscó generar respuestas puntuales a través de proyectos específicos en los que participaban especialistas de muy diversas áreas. Ambas corrientes generaron resultados que influyeron en la consolidación de proyectos importantes e incrementando la productividad en los años siguientes.

De particular relevancia fue la creación, en 1981, de la Especialización en Estadística Aplicada, que surgió en respuesta a la necesidad de contar con un programa de estadística aplicado a otras áreas del conocimiento, en el que no se requería de una sólida formación matemática para poder cursarlo.

Actualmente el IIMAS forma parte de los 19 institutos que pertenecen al Subsistema de la Investigación Cientifica de la Universidad Nacional Autónoma de México; se agrupa en el Área de las Ciencias Físico Matemáticas y de las Ingenierías, y está organizado en seis departamentos académicos. El Instituto da gran 
importancia a su labor formativa en licenciatura y participa de manera central en los posgrados de: Ciencias Matemáticas, Especialización en Estadística Aplicada; Ciencia e Ingeniería de la Computación; Ingeniería y Ciencias de la Tierra. Asimismo, apoya la formación de recursos humanos a través de cursos, talleres, seminarios, proyectos de investigación y educación continua, así como en la difusión del conocimiento.

Han sido directores del Instituto: Dr. Tomás Garza Hernández (1976-1982); Dr. Alejandro Velasco Levy (1982-1984); Dr. José Luis Abréu León (1984-1988); Dr. Ignacio Méndez Ramírez (1988-1996); Dr. Ismael Herrera Revilla (1996-2000); Dr. Federico J. O'Reilly Togno (2000-2004); Dr. Demetrio Fabián García Nocetti (2004-2012) y a partir del 24 de abril de 2012 el Instituto está dirigido por el Dr. Héctor Benítez Pérez. ${ }^{2}$

\section{La Biblioteca IIMAs}

\section{Antecedentes}

El edificio del IIMAS estuvo inicialmente proyectado dentro del plano del conjunto de la construcción de Ciudad Universitaria como área habitacional para estudiantes (propuesta que no se llevó a cabo), dándole un uso distinto al propuesto. El proyecto y realización del inmueble estuvo a cargo de los arquitectos Manuel Martínez y Enrique Carral Icaza. Durante los primeros años de albergar al IIMAS, la Biblioteca ocupaba aproximadamente la mitad del ala sur del segundo piso, espacio que al correr de los años resultó no sólo insuficiente sino incluso inadecuado, ya que por el peso de los libros el edificio empezó a tener un asentamiento considerable hacia la zona oriente. En consecuencia, la Dirección General de Obras de la UNAM (DGO-UNAM) revisó la estructura y recomendó reducir el peso en esa superficie y, dada

2 Universidad Nacional Autónoma de México. Instituto de Investigaciones en Matemáticas Aplicadas y en Sistemas. 35 años en la vida del Instituto de Investigaciones en Matemáticas Aplicadas y en Sistemas [en línea]. Video archivos de reuniones emblemáticas IIMAs. Marzo 2012. <http://www.iimas.unam.mx/ iimas/pagina/es/141/reuniones-academicas-en-linea-y-archivo-de-videograbaciones $>$ [Consulta: septiembre 2012]. la falta de espacio y el riesgo de falla estructural del piso, se decidió asignar una nueva área a la Biblioteca en el ala norte del mismo piso del edificio, donde fue colocada la Hemeroteca; esto provocó cambios en los procedimientos de servicios al público y que la Biblioteca funcionara en dos áreas separadas en los extremos del segundo piso del edificio original. No obstante esta distribución se continuaba con sobrepeso, lo que hacía urgente y necesaria la construcción de un espacio adecuado para la Biblioteca.

Durante 1989, la Mtra. Blanca Castañón Moreno, jefa de la Biblioteca, tuvo varias reuniones con los doctores Ignacio Méndez Ramírez y Jaime Jiménez Guzmán, director y secretario académico -respectivamente- del IIMAS, para plantear la necesidad de un nuevo inmueble para la Biblioteca. Posteriormente, se presentó al Comité de Biblioteca del Instituto la idea de construir un nuevo edificio; después de trabajar en coordinación con la DGO-UNAM en diferentes anteproyectos de un nuevo espacio para la $\mathrm{Bi}$ blioteca, y de negociaciones, evaluaciones y presupuestos, se logró la aprobación del proyecto del nuevo edificio bajo la dirección del arquitecto Arturo Ayala Gastelum.

Así, para diciembre de 1992 se colocó la primera piedra del edificio anexo donde se ubican el auditorio, los posgrados y la biblioteca, siendo inaugurado el 24 de septiembre de 1996 por el rector Dr. José Sarukhán Kérmez. ${ }^{3}$

\section{Situación actual}

La Biblioteca del Instituto de Investigaciones en Matemáticas Aplicadas y en Sistemas (Biblioteca IIMAS) es considerada como una de las mejores bibliotecas especializadas en el área de las matemáticas aplicadas y computación; y como parte del Sistema Bibliotecario de la UNAM ofrece acceso a los recursos impresos y electrónicos que la Universidad brinda a la comunidad universitaria, incluyendo además aquellos de acceso libre o los generados por la propia UNAM, logrando de esta manera que a través de los servicios bibliotecarios

\footnotetext{
$3 \times$ Aniversario del edificio anexo que alberga la biblioteca, el auditorio del IIMAS y los posgrados en que participa [cartel]. Diseño Gráfico: DGC. Vanessa Gil Tejeda. UnAM, Unidad de Publicaciones y Difusión-IIMAs, Octubre 2006.
} 

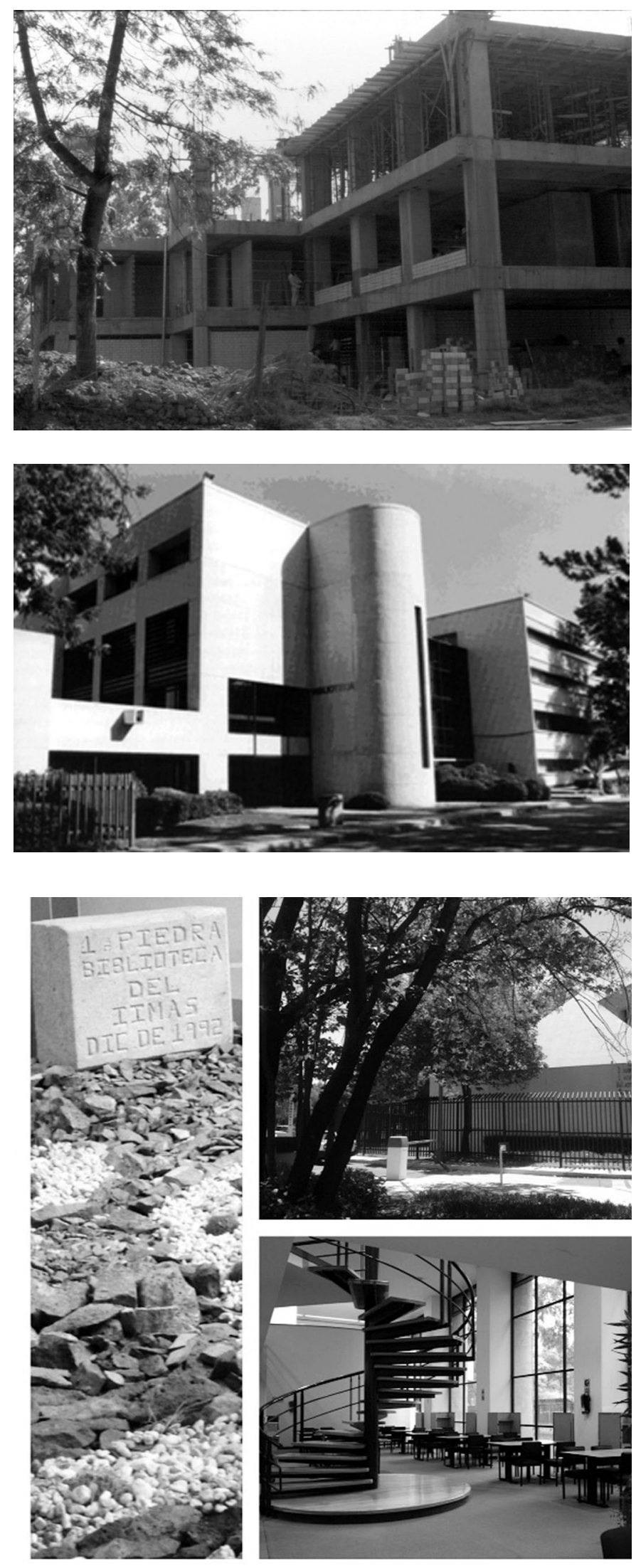

Fotos cortesía del Archivo fotográfico e histórico del IIMAS.

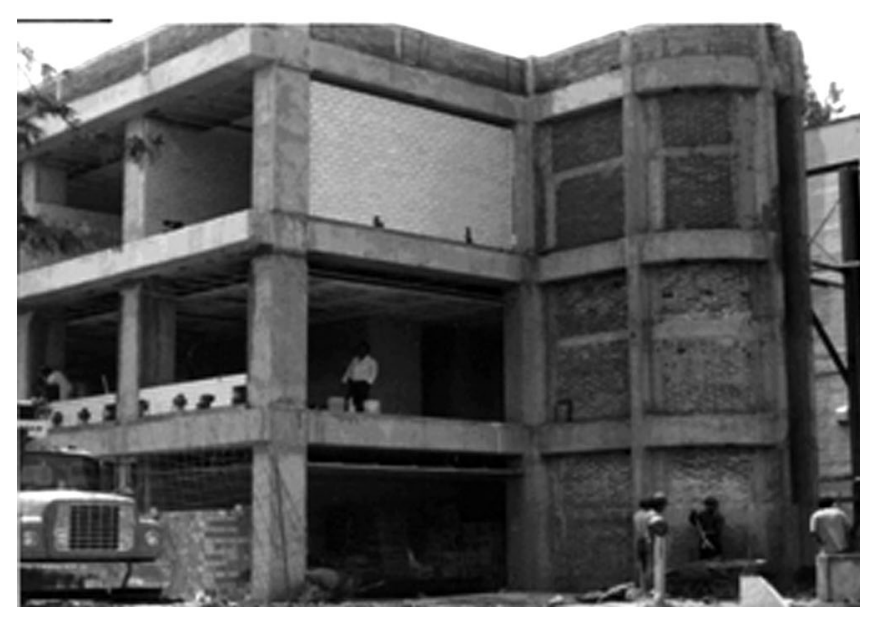

y de información se cubran las necesidades informativas de la comunidad del IIMAS, de los profesores y estudiantes de los posgrados adscritos al Instituto y de usuarios de otras entidades académicas y administrativas ubicadas dentro y fuera del campus universitario.

\section{Misión}

De acuerdo al Reglamento de la Biblioteca del Instituto de Investigaciones en Matemáticas Aplicadas y en Sistemas, ésta tiene como misión "ser una entidad dinámica y de vanguardia que incida de manera decisiva, oportuna y eficiente en los procesos de generación, transmisión y difusión de conocimiento en el país, a través de sus recursos y servicios".

\section{Objetivo}

La Biblioteca IIMAS tiene como objetivo principal "satisfacer las necesidades de información del personal académico del Instituto, de los profesores y estudiantes de los posgrados en que participa el IIMAS y en general a la comunidad universitaria, en las áreas del conocimiento que se cultivan en el Instituto". 5

\footnotetext{
De la misión, objetivo y funciones. En: Reglamento de la Biblioteca del Instituto de Investigaciones en Matemáticas Aplicadas y en Sistemas, Capítulo II, Art. 4, UNAM, Instituto de Investigaciones en Matemáticas Aplicadas y Sistemas, p. 1. Idem.
} 


\section{Organización}

La Biblioteca se encuentra organizada por una jefatura de la que dependen tres áreas y cuenta con el apoyo de la Comisión de Biblioteca como un órgano consultivo de la Dirección del Instituto.

En junio de 2001 la Biblioteca modificó su reglamento interno y reorganizó su estructura orgánica. Anteriormente existian tres áreas, dos de ellas dedicadas a la adquisición de libros y revistas y la tercera destinada a realizar actividades de circulación de los recursos de información. Con la reorganización se reagrupan las actividades de adquisición de materiales en una sola área (Servicios Técnicos), continúa con sus actividades y redefiniendo sus funciones el área de Servicios al Público y se crea una nueva, Servicios Especializados, que se encarga principalmente de difundir servicios y recursos digitales especializados y de la diseminación selectiva de información.

Cada área en particular tiene funciones especificas, así como servicios básicos y especializados, que en su conjunto coadyuvan en el cumplimiento del objetivo de la Biblioteca apoyando a los seis departamentos académicos que conforman el Instituto, con un promedio de 60 investigadores por año y alrededor de 40 técnicos académicos.

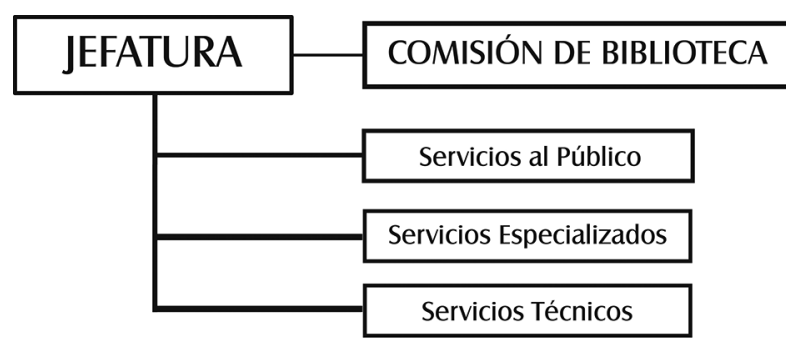

Departamentos académicos a los que atiende la Biblioteca IIMAS

Organización interna de la Biblioteca y departamentos académicos a los que atiende.

\section{Instalaciones y espacios}

El inmueble que actualmente ocupa la Biblioteca tiene una superficie construida de $1107 \mathrm{~m}^{2}$ en dos niveles. Su diseño es sobrio, dentro del concepto moderno de funcionalidad; su construcción está dispuesta de tal manera que recibe luz natural abundante a través de múltiples ventanas, que por sus cualidades estéticas dan como resultado un espacio confortable y apropiado para la realización de las tareas académicas que apoyan a la docencia e investigación.

En la planta alta se encuentra la entrada principal que conduce directamente al área de catálogos electrónicos, al acervo general, a obras de consulta y tesis; de igual manera, se encuentra el módulo de atención a usuarios, áreas de lectura con capacidad para 16 personas, una sala de juntas, una pequeña área de novedades bibliográficas y oficinas para uso de la administración de la propia Biblioteca (Jefatura, Servicios Especializados y Servicios al Público). En el otro nivel y bajando por las escaleras internas se ingresa a la colección de publicaciones periódicas, una sala general colectiva con capacidad para 53 usuarios, además de una área de lectura informal de 16 lugares y una área de exhibición de las revistas más recientes distribuidas por áreas temáticas. Como un servicio adicional

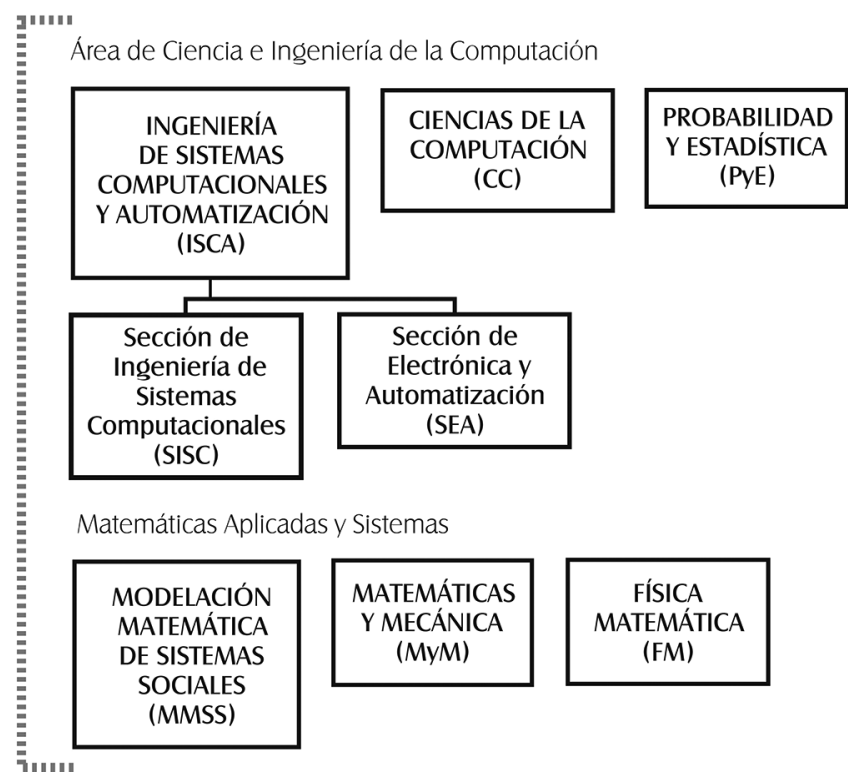




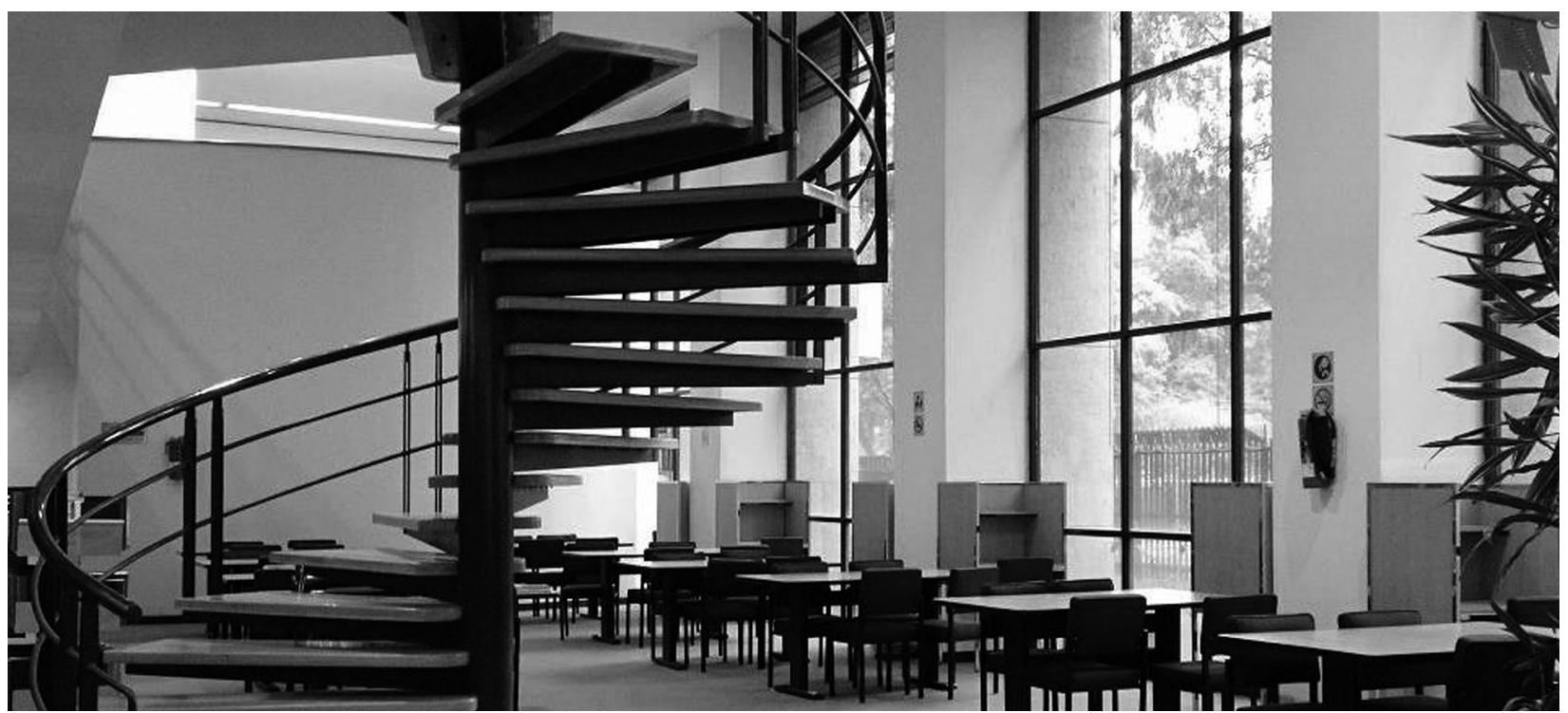

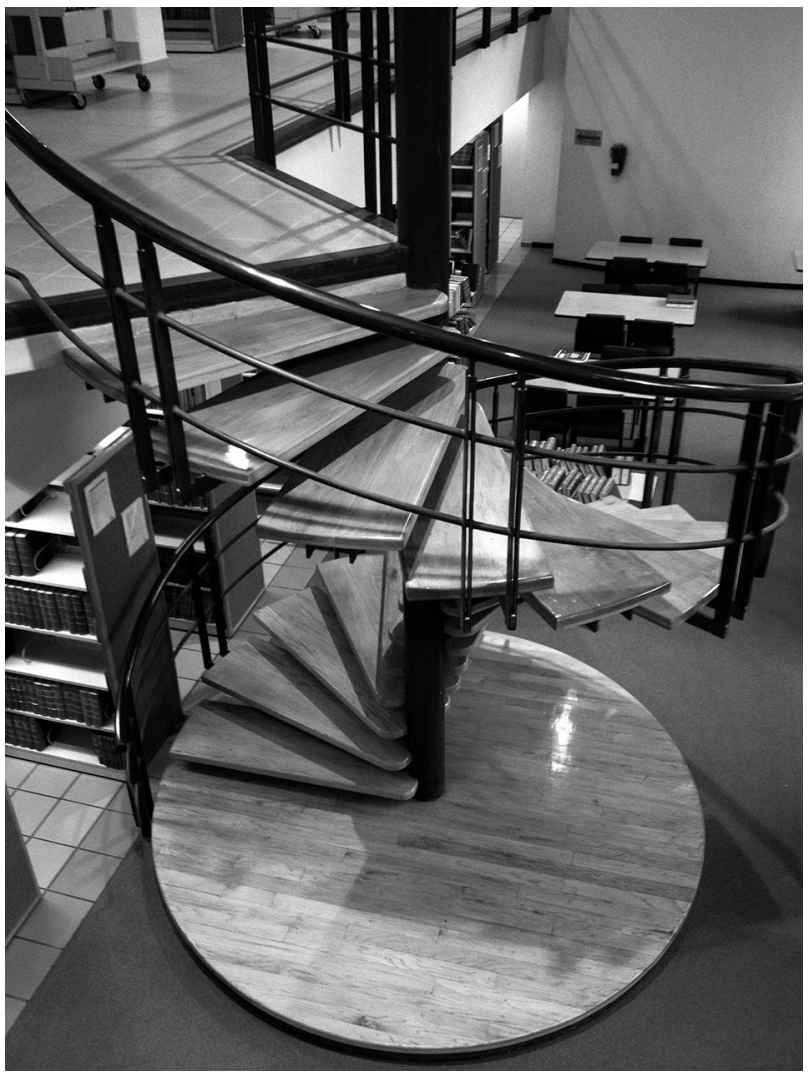

se encuentran los cubículos de estudio como apoyo a la realización de trabajos en grupo, disponibles para alumnos y profesores pertenecientes a los programas de posgrado con sede en el Instituto, y -finalmentese ubica el área de Servicios Técnicos, en donde se llevan a cabo tareas de adquisición y proceso físico del material bibliohemerográfico.

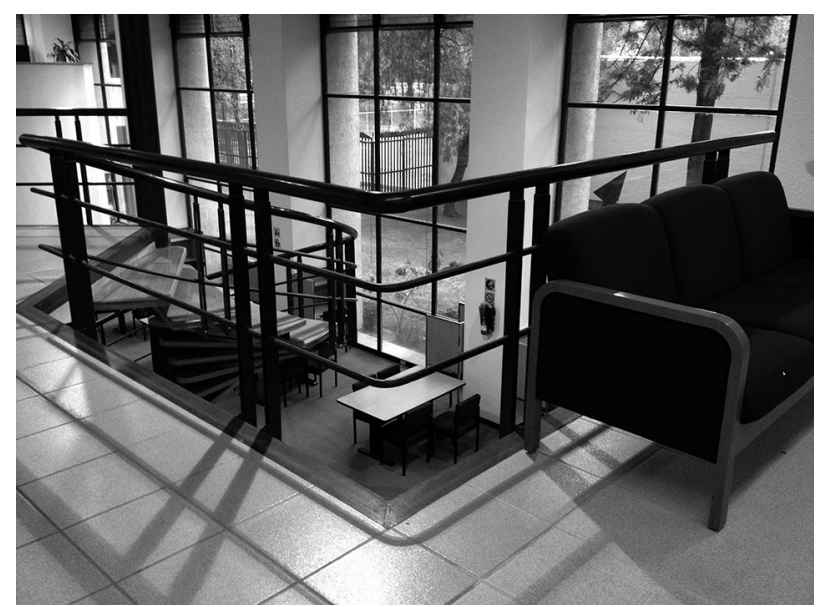

Todas las áreas están habilitadas con red inalámbrica, tanto para la RIU (Red Inalámbrica Universitaria) como la red local del IIMAS.

\section{Colecciones}

La Biblioteca cuenta con colecciones impresas, multimedia y electrónicas. Dentro de los dos primeros grupos se cuenta con 29752 volúmenes de libros (incluyendo obras de consulta), 689 tesis de maestría, doctorado y especialización y 937 títulos de publicaciones periódicas vigentes y no vigentes. Además, se cuenta con 4070 unidades de multimedia en diversos formatos (CD-ROM, diskettes, microfilms, microfichas, diapositivas, videograbaciones, etcétera). Actualmente, la colección bibliográfica impresa se encuentra distribuida en las siguientes temáticas principales: 
Tabla 1. Temas principales de la colección

\begin{tabular}{|c|c|c|c|}
\hline $\begin{array}{l}\text { Clase / } \\
\text { Subclase }\end{array}$ & Tema & $\begin{array}{l}\text { Clase / } \\
\text { Subclase }\end{array}$ & Tema \\
\hline HA & $\begin{array}{l}\text { Estadística } \\
\text { - Teoría y método de la estadística en } \\
\text { ciencias sociales } \\
\text { - Datos estadísticos }\end{array}$ & \multirow[t]{2}{*}{ Qc } & \multirow{2}{*}{$\begin{array}{l}\text { Física } \\
\text { - Pesos y medidas } \\
\text { - Mecánica descriptiva y experimental } \\
\text { - Acústica. Sonido } \\
\text { - Termodinámica } \\
\text { - Óptica } \\
\text { - Geofísica }\end{array}$} \\
\hline \multirow{2}{*}{ HB } & \multirow{2}{*}{$\begin{array}{l}\text { Teoría económica. Demografía } \\
\text { - La economía como ciencia } \\
\text { - Métodos cuantitativos incluyendo } \\
\text { econometría, análisis y teoría de } \\
\text { juegos }\end{array}$} & & \\
\hline & & \multirow[b]{2}{*}{$T$} & \multirow{2}{*}{$\begin{array}{l}\text { Tecnología } \\
\text { - Matemáticas aplicadas. Métodos } \\
\text { cuantitativos } \\
\text { - Análisis de sistemas } \\
\text { - Tecnología de la información }\end{array}$} \\
\hline \multirow{2}{*}{ HD } & $\begin{array}{l}\text { Industrias. Uso de la tierra. Trabajo } \\
\text { • Innovaciones tecnológicas. } \\
\text { Automatización }\end{array}$ & & \\
\hline & $\begin{array}{l}\text { - Comportamiento organizacional } \\
\text { - Grupos de trabajo }\end{array}$ & \multirow{3}{*}{ TA } & \multirow{3}{*}{$\begin{array}{l}\text { Ingeniería. Ingeniería Civil } \\
\text { - Bioingeniería } \\
\text { - Instrumentos, medidores, } \\
\text { instrumentación industrial } \\
\text { - Ingeniería humana } \\
\text { - Ingeniería de sistemas } \\
\text { - Mecánica aplicada } \\
\text { - Ingeniería acústica }\end{array}$} \\
\hline HF & Comercio & & \\
\hline HG & $\begin{array}{l}\text { Finanzas } \\
\text { - Liquidez } \\
\text { - Procesamiento de datos } \\
\text { - Seguros }\end{array}$ & & \\
\hline Q & $\begin{array}{l}\text { Ciencias } \\
\text { - Cibernética } \\
\text { - Teoría de la información }\end{array}$ & \multirow[t]{2}{*}{$\mathrm{TJ}$} & \multirow{2}{*}{$\begin{array}{l}\text { Ingeniería Mecánica } \\
\text { - Dinámica } \\
\text { - Dispositivos mecánicos. Autómatas } \\
\text { - Robots } \\
\text { - Construcción de máquinas }\end{array}$} \\
\hline & \multirow{2}{*}{$\begin{array}{l}\text { Matemáticas } \\
\text { - Tablas } \\
\text { - Instrumentos y máquinas } \\
\text { - Ordenadores electrónicos. Informática } \\
\text { - Software, aplicaciones } \\
\text { - Matemáticas elementales. Aritmética } \\
\text { - Álgebra } \\
\text { - Probabilidad. Estadística matemática } \\
\text { - Geometría, trigonometría y topología }\end{array}$} & & \\
\hline QA & & TK & $\begin{array}{l}\text { Ingeniería Eléctrica. Electrónica. } \\
\text { Ingeniería Nuclear } \\
\text { - Telecomunicación. Telegrafía, radar, } \\
\text { televisión } \\
\text { - Ingeniería electrónica } \\
\text { - Ingeniería de hardware }\end{array}$ \\
\hline QB & $\begin{array}{l}\text { Astronomía } \\
\text { - Teoría de la astronomía y mecánica } \\
\text { celeste } \\
\text { - Astrofísica }\end{array}$ & & \\
\hline
\end{tabular}

Para facilitar la consulta del acervo, la Biblioteca funciona bajo el concepto de estantería abierta, con una colección conformada principalmente por libros en las áreas de matemáticas aplicadas, estadística, ciencia e ingeniería de la computación y sistemas sociales; así como fascículos de revistas científicas especializadas. 

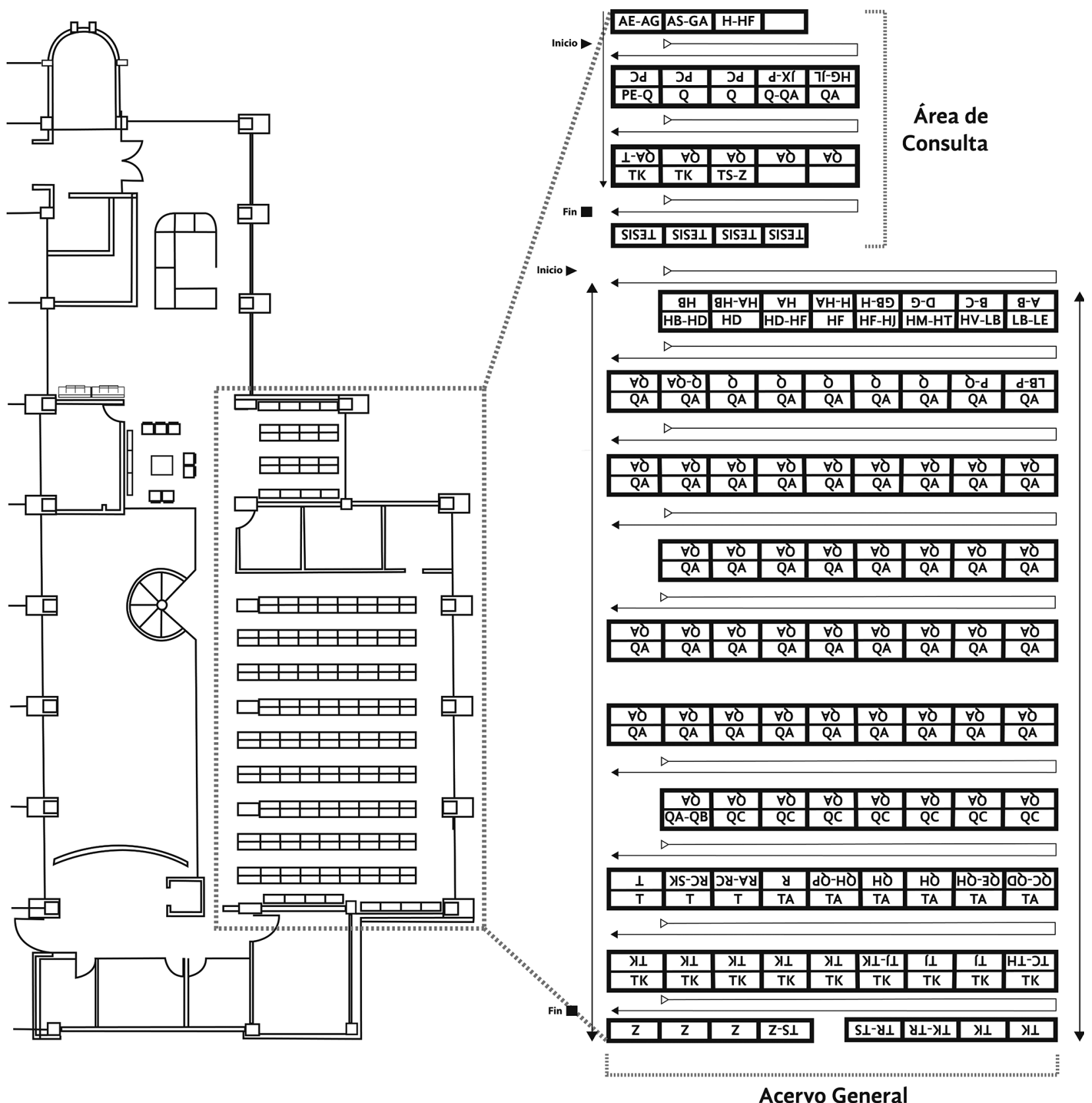

\section{Acervo General \\ Plano arquitectónico, planta alta, distribución de acervo bibliográfico. \\ Diseño Gráfico: DCG. Vanessa Gil Tejeda, Unidad de Publicaciones y Difusión-IIMAS, unam. Octubre 2012.}

Con relación a las colecciones electrónicas o digitales, la Biblioteca IIMAS ha adquirido libros electrónicos desde el año 2001. Las primeras compras se hicieron a través de proveedores nacionales que en ese momento representaban a editoriales extranjeras. En los primeros ocho años las compras fueron incipientes y no llegaron a más de 100 títulos; para el año de 2009, la biblioteca del Instituto junto con las del Instituto de Matemáticas y la Facultad de Ciencias, con el apoyo de la Subdirección de Servicios de Información Especializada de la DGB, iniciaron la compra conjunta de algunas colecciones de libros electrónicos de la editorial Springer. Dada la cobertura de las colecciones y el costo, se invitó a participar a otras bibliotecas y dependencias académicas de la UnAm en esta compra, la cual se ha llevado a cabo también en los años de 2010 a 2012. 
Hasta este año, la Biblioteca ha participado en la compra conjunta de 13600 títulos de las editoriales Springer, Wiley, SIAM, Cambridge University Press, Oxford University Press, Elsevier y World Scientific Publishing. Estas compras han sido favorables para la Universidad, ya que los materiales se han adquirido a perpetuidad, con acceso multiusuario y concurrencias ilimitadas para toda la UnAm. En la página web de la biblioteca (www.iimas.unam.mx/biblio) se ofrece acceso a más de 830 títulos en las áreas de matemáticas, computación, probabilidad y estadística y ciencias sociales.

De las revistas electrónicas, la Biblioteca ha adquirido un número importante de títulos bajo el esquema título impreso + título electrónico (IEEE, ACM, IOP, SIAM, principalmente); este esquema ha ido evolucionando desde vender la suscripción impresa y dar el acceso electrónico gratuito hasta vender el acceso electrónico y por un porcentaje ofrecer la versión impresa.

Hasta el año 2009 la Biblioteca IIMAS tramitaba directamente las suscripciones a sus revistas a través de un gestor (Swets) y a partir del año 2010 el Departamento de Suscripciones de la DGB centraliza dichas suscripciones y es quien ahora tramita las suscripciones y accesos a las versiones electrónicas de las revistas.

Uno de los beneficios importantes que ha conseguido la UNAM en la gestión de la compra de revistas ha sido la negociación de las licencias de acceso, en donde se ha logrado que dichos accesos sean para toda la Universidad y de ahí la posibilidad de que la Biblioteca IIMAS haya incorporado a su página web títulos que no ha adquirido de manera directa y actualmente cuente con más de 470 títulos de revistas.

\section{Usuarios}

Como ya se indicó, la Biblioteca tiene como objetivo principal satisfacer las necesidades de información del personal académico, de los profesores y los estudiantes de los posgrados en que participa el Instituto y en general de la comunidad universitaria, en las áreas temáticas correspondientes:
- Matemáticas Aplicadas y Sistemas

- Ciencia e Ingeniería de la Computación

En los niveles de posgrado se atienden, conjuntamente con las bibliotecas de facultades (Facultad de Ciencias y Facultad de Ingeniería) y el Instituto de Matemáticas, las necesidades de información de los participantes en los programas de posgrado del Área de las Ciencias Físico-Matemáticas y de las Ingenierías:

- Ciencias Matemáticas

- Ciencia e Ingeniería de la Computación

- Ciencias de la Tierra

- Ingeniería

De manera específica, la Biblioteca IIMAS atiende anualmente un promedio de 10300 usuarios aproximadamente (alumnos y profesores de nivel medio superior, licenciatura y Posgrado) interesados en las áreas de Actuaría, Ciencias de la Computación, Ciencias de la Tierra, Física, Astrofísica, Matemáticas, Ingeniería de Materiales, entre otras líneas de estudio e investigación.

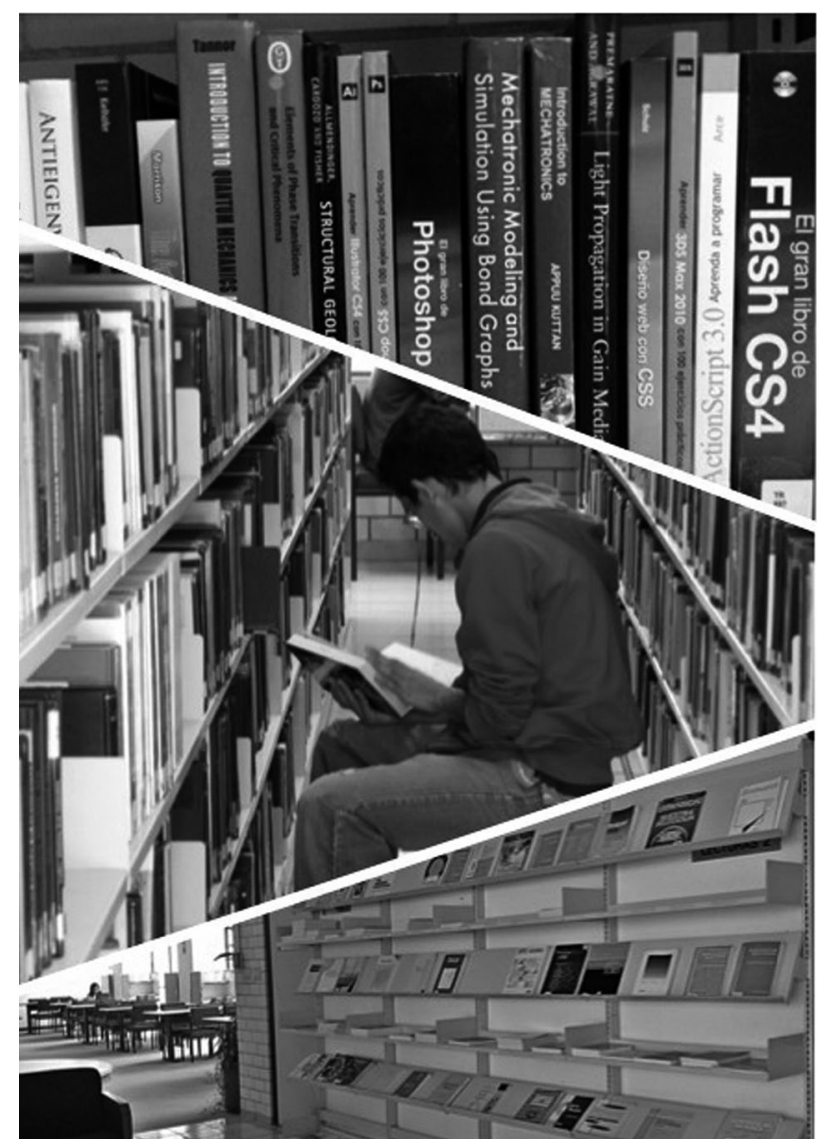




\section{Servicios bibliotecarios}

La Biblioteca IIMAS ofrece diversos servicios bibliotecarios (básicos y especializados) para llevar a buen fin sus objetivos y -por ende- como apoyo a las actividades que desarrolla la comunidad académica del propio Instituto.

Tabla 2. Servicios bibliotecarios.

\begin{tabular}{|l|l|}
\hline Servicios básicos & \multicolumn{1}{c}{$\begin{array}{c}\text { Servicios } \\
\text { especializados }\end{array}$} \\
\hline - Préstamo en & - Análisis de citas \\
sala, a domicilio e & - Diseminación selectiva \\
interbibliotecario & de información \\
- Préstamo de cubículos & - Obtención y suministro \\
de estudio & de documentos \\
- Orientación e & \\
información a usuarios & \\
\hline Reserva de material & \\
documental & \\
\hline Boletín de nuevas & \\
adquisiciones y alerta & \\
bibliográfico &
\end{tabular}

A través de estos servicios y de los recursos con los que se cuenta, la biblioteca -con apoyo de otras bibliotecas afines- ha podido atender las necesidades de información que día con día exige la comunidad, y no sólo a nivel local sino también dentro y fuera del campus universitario. De ahí que el préstamo interbibliotecario (PIB) ha sido uno de los servicios prioritarios y con mayor alcance en la obtención y suministro de documentos. En este sentido, la Biblioteca IIMAS mantiene convenios con más de 100 instituciones, muchas de ellas pertenecientes a los subsistemas de Licenciatura y Posgrado, Investigación Científica e Investigación en Humanidades de la UNAM, así como con instituciones de educación superior, centros de investigación y dependencias gubernamentales dentro y fuera de la zona metropolitana, tales como: el Banco de México, la Facultad Latinoamericana de Ciencias Sociales-FLACSO, el Instituto Tecnológico Autónomo de México-ITAM, el Instituto Politécnico Nacional (IPN), Petróleos Mexicanos (PEMEX), el Instituto Nacional de Investigaciones
Nucleares (ININ), el Centro de Investigación en Matemáticas (CIMAT-Guanajuato), el Instituto Nacional de Astrofísica, Óptica y Electrónica (INAOE-Puebla), entre otros.

Por otra parte, el servicio de gestión de documentos en el extranjero ha permitido seguir atendiendo las demandas de información de los investigadores a través de The British Library; además, la Biblioteca IIMAS es participante del programa de Préstamo Interbibliotecario Transnacional México-E.U.A., que tiene como propósito fomentar la cooperación para compartir recursos y apoyar la recuperación de información. Al respecto cabe mencionar que gracias a este recurso los documentos que se recuperan son obtenidos sin costo alguno, lo que ha favorecido el presupuesto destinado para dicha actividad.

\section{Implementación del Sistema Integral para la Automatización de Bibliotecas (ALEPH)}

La Biblioteca IIMAS inicia la implementación del sistema ALEPH el 5 de junio del año 2000 con la instalación del módulo de Circulación, el cual permite administrar el préstamo a domicilio; desde ese momento y hasta la fecha la Biblioteca ha participado activamente en la adopción de este sistema. En este mismo año, el personal revisó el módulo de Adquisiciones y se determinó que aún dicho módulo no contaba con las funciones y aplicaciones que en ese momento se requerían, y fue hasta mayo de 2007, ya con algunas mejoras, que se instala y se empieza su uso aunque de manera parcial. Por otra parte, el módulo de Catalogación se implementó en 2001 con el propósito de realizar los cargos remotos de los libros adquiridos por la Biblioteca y con esto agilizar la puesta en circulación de los materiales.

En 2004 se decide crear el catálogo local de las tesis presentadas por los alumnos de la Especialización en Estadística Aplicada y de los posgrados en que participa el IIMAS, por lo tanto se hizo imprescindible que se habilitara el módulo de Catalogación para registrar en TESIUNAM estas tesis y crear el catálogo TESIIMAS. El módulo Seriadas inicialmente se instaló en 2003 pero fue hasta el 2009 cuando se empezó a utilizar regularmente para apoyar las actividades del inventario de revistas. En 
2010, la Dirección General de Bibliotecas llevó a cabo una prueba piloto para implementar en las bibliotecas del sistema el módulo de Préstamo Interbibliotecario; la Biblioteca IIMAS participó en dicha prueba y a partir de ese momento queda funcionando el módulo; también en este año se aloja la aplicación de Avisos de cortesía en el módulo de Circulación. El módulo de ALEPH que se ha instalado más recientemente (2011) es el de Renovación, que permite que los usuarios puedan realizar directa y personalmente las renovaciones de sus préstamos a domicilio a través de la página web de la biblioteca, así como consultar de manera autónoma su historial de préstamos, incluyendo los vigentes y próximos a vencer.

\section{Proyectos internos y participación en otros programas}

En los últimos once años la Biblioteca IIMAs ha emprendido y participado en diferentes actividades que le han permitido mejorar sus servicios e incidir en algunos de los avances del sistema bibliotecario de la Universidad.

En el año 2001 la Subdirección Técnica de la Dirección General de Bibliotecas (ST-DGB) inició su participación en el Proyecto de Control de Autoridades Temáticas en donde participaban, entre otras, las bibliotecas de instituciones como El Colegio de México, el ITAM, la Universidad Iberoamericana, la Universidad de las Américas (Cholula, Puebla), etcétera. Particularmente, la Biblioteca IIMAS colaboró de 2002 a 2003 directamente con la ST-DGB en la creación de algunas autoridades temáticas para las áreas de matemáticas, computación y estadística. De manera simultánea y en coordinación con la Unidad de Publicaciones y Difusión del IIMAS, se inició el Programa de Catalogación en la Fuente como una actividad interna con el objetivo de propiciar la normalización de los registros y agilizar el procesamiento técnico de los documentos; es así que se han elaborado fichas catalográficas de cerca de 10 títulos de libros publicados por los diferentes departamentos académicos que integran el Instituto.

De 2002 a 2006 la Biblioteca formó parte de dos instancias consultivas de la Dirección General de Bibliotecas: Comité del Libro Electrónico y Comité Evaluador de Recursos de Información Electrónica, cuya finalidad era la de establecer lineamientos y criterios para el desarrollo de colecciones electrónicas o digitales que apoyaran las actividades sustantivas de la Universidad; estos comités desaparecieron en $\mathbf{2 0 0 8}$ pero los resultados de sus acciones han permeado en las actividades que actualmente realiza la UNAM para la adquisición de recursos de información en este tipo de formatos.

En febrero de 2003 se inició un análisis de la capacidad y distribución de los espacios dedicados a las colecciones de materiales impresos dentro de la biblioteca solicitando a la DGB un estudio para proyectar a diez años las necesidades de espacio y mobiliario. Los resultados de dicho estudio dieron pauta para que en junio del mismo año se solicitara a BIBLIOMODEL (empresa dedicada al diseño, planeación de espacios e instalación de mobiliario para bibliotecas con certificación de Iso 9001) una propuesta gráfica y funcional que permitiera una mejor redistribución de la estantería tomando en consideración la información del estudio realizado por la DGB. Es así que en 2004 se logra ampliar el área de la colección de libros y en 2010 la de revistas. El estudio y la propuesta siguen vigentes y conforme sea necesario se irá incrementado la estantería y reacomodando los espacios, aunque el ritmo de crecimiento de las colecciones ha disminuido por la adquisición de libros y revistas en formato digital.

A principios de 2005 se conformó de manera informal la llamada Red de Bibliotecas de Matemáticas, en donde participan el Instituto de Matemáticas, la Facultad de Ciencias y el IIMAs. El objetivo principal de esta red es detectar, analizar y dar solución a los problemas que pudiera presentar la comunidad académica de docentes y alumnos de la Maestría y Doctorado en Ciencias Matemáticas y de la Especialización en Estadística Aplicada en cuanto al uso de los servicios bibliotecarios y los recursos de información.

Otro proyecto de suma importancia fue el primer inventario de libros que se inició en junio de 2005 y concluyó en todas sus etapas en mayo de 2006. Esta actividad representó un reto para el personal de la biblioteca, por un lado porque no se tenía experiencia previa y, por otra parte, para poder planear dicho inventario fue necesario diseñar y realizar una prueba piloto que fue propuesta $e$ 
implementada por investigadores del Departamento de Probabilidad y Estadística del IIMAS. Las etapas del inventario fueron el cotejo físico, identificación y corrección de errores, reproceso físico e incorporación del material a la estantería, actividades que concluyeron de manera exitosa de acuerdo a las estimaciones de la prueba piloto.

En relación al inventario de publicaciones periódicas, en 1997 se realizó un conteo para determinar el número de fascículos que se tenían hasta ese momento; sin embargo, no existió ninguna intención de que esta información pudiera ser utilizada para iniciar un inventario. En el año 2003 se llevó a cabo un intento formal con un avance de cerca del $10 \%$ pero no se continuó debido a las cargas de trabajo que implicaban para realizar esta actividad de manera manual y en formatos impresos. En septiembre de 2009, nuevamente se inicia el inventario, pero ahora ya con el apoyo del módulo de Seriadas del sistema ALEPH, y para septiembre de 2012 se tiene un avance de cerca de 95\%, tanto en el cotejo físico de los fascículos como en la captura de información en dicho módulo.

\section{Las nuevas tecnologías en la Biblioteca IIMAS}

\section{Página Web}

La actual página web de la biblioteca se encuentra en su cuarta versión. Ha pasado de ser meros listados de revistas impresas elaborados en lenguaje HTML a un sistema dinámico y complejo que incluye el acceso a bases de datos, libros y revistas digitales, catálogos de recursos de información impresos y electrónicos, boletines, así como a formularios para solicitar varios de los servicios que se ofrecen y para la consulta de préstamos y la renovación de préstamos a domicilio vía web. Además, con el Carrusel de noticias, la biblioteca ofrece otro canal más de comunicación con sus comunidades.

\section{Presencia en las redes sociales}

A mediados de 2011 la biblioteca se introdujo en el uso de las redes sociales en Internet a través de Facebook y Twitter para difundir información sobre las

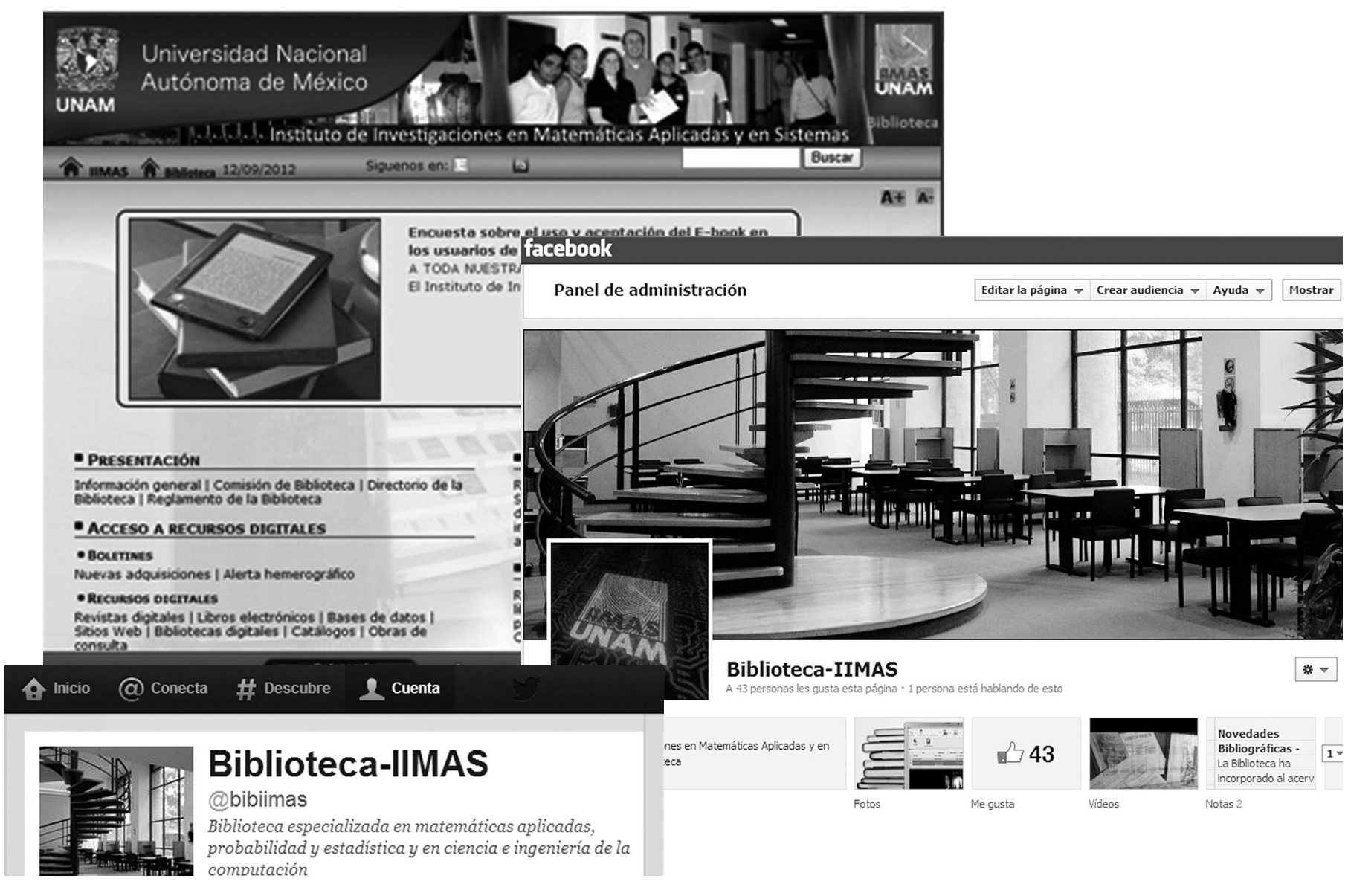


actividades de la biblioteca, proveer el acceso a recursos de información impresos y electrónicos y ofrecer cualquier dato que se considere relevante para la comunidad académica del Instituto y para los profesores y alumnos de los posgrados asociados al IIMAS; en la página web de la biblioteca aparecen los íconos que ofrecen los enlaces para acceder a estas redes.

Para la Biblioteca IIMAS formar parte de las redes sociales como una opción alterna de difusión resulta de gran importancia, así como poder estar presente en un espacio mucho más interactivo, colaborativo y guiado por las necesidades de la comunidad de usuarios a la que sirve. El objetivo es incluir al usuario en una participación activa en donde manifiesten sugerencias y necesidades informativas; de esta manera contribuirá a que los servicios bibliotecarios se estén actualizando y renovando constantemente para beneficio de la comunidad IIMAs y para el público en general.

\section{Perspectivas}

Hoy en día las bibliotecas especializadas están experimentando diversos cambios de paradigmas que las han ido llevando a replantear su quehacer y su posición dentro del contexto en que funcionan.

En tan solo una generación se han manifestado cambios en la comunicación académica, lo que a su vez ha transformado inevitablemente la naturaleza de la obtención y suministro de documentos, por lo que la Biblioteca debe gestionar con las partes implicadas (autores y bibliotecas) para ofrecer un servicio con mayor alcance. Es en este marco de referencia en donde hay que buscar nuevos modelos de negociación (redes y asociación de bibliotecas), ya que la optimización de recursos económicos es una de las razones por las que es indispensable realizar tareas de cooperación entre bibliotecas y -por ende- buscar alternativas que coadyuven en la obtención y suministro de documen- tos, por lo que el objetivo es negociar y establecer acuerdos de cooperación con otros programas nacionales e internacionales.

De igual manera, el movimiento Open Access, o de acceso abierto a la producción científica, ha experimentado en los últimos años un avance significativo, por lo que es importante revisar y pensar en este tema como una opción más para ampliar y mejorar el servicio de suministro de documentos que se ofrece actualmente a la comunidad académica del Instituto.

La Biblioteca deberá crear usuarios autosuficientes capaces de manejar herramientas que les permitan localizar e identificar información relevante para sus actividades. En esta tarea es necesario desarrollar e implementar un programa de Formación de usuarios que contemple acciones tales como los diagnósticos, pláticas, talleres, cursos, seguimientos y evaluaciones.

Las colecciones de documentos digitales o electrónicos seguirán teniendo preponderancia en la adquisición de recursos de información para apoyar las actividades sustantivas del Instituto, esto llevará a un replanteamiento de la plataforma que actualmente se utiliza para el registro y acceso de estos recursos, considerando fuertemente el uso de un administrador de contenidos de código abierto.

Las ideas y propuestas planteadas hacen necesario analizar la funcionalidad de la Biblioteca. A través de un diagnóstico objetivo se deberá determinar si existe la necesidad de crear nuevos servicios o rediseñar o reorganizar aquellos que no estén teniendo un impacto importante para el conjunto de usuarios.

El posicionamiento que logre la Biblioteca dentro del IIMAS, de la Universidad y en el exterior estará sustentado en el impacto que sus recursos y servicios tengan en las comunidades a las que sirve. $\cos$ 